\title{
Comparison between Homemade Toothpaste and Commercial Tooth Paste in Plaque Removal of Children's in Udaipur City Rajasthan
}

\author{
Dr.Shashank Chaudhari ${ }^{1}$, Dr.Ruchi Arora ${ }^{2}$, Dr. Subha Dogra ${ }^{3}$, \\ Dr.Sonal Kothari ${ }^{4}$ \\ (Darshan dental college, Udaipur, Rajasthan, India)
}

\begin{abstract}
Aims: The aim of the study is to determine efficacy of homemade toothpaste in plaque removal of children in Udaipur city.

Material and method:- 30 children including boys and girls with the age of 8 and 12 years were selected for this study and randomly divided into 2 groups. Group A: children who were prepared to brush their teeth by homemade toothpaste. Group B: children who were prepared to brush their teeth by commercial toothpaste. The evaluation of score was done on the entire dentition or on selected teeth. Only the plaque of the cervical third of the tooth was evaluated by using disclosing solution.

Result: On intra-group comparison, a significant reduction in plaque scores was obtained in the first week itself.

Conclusions: The result of this study has given useful information about the positive effect of homemade toothpaste on decreasing plaque score. It is suggested that the use of the homemade toothpaste containing coconut could be useful aid to obtaining a significant reduction for plaque index compared to the commercial toothpaste.
\end{abstract}

Key words: Homemade toothpaste and commercial toothpaste, sillness and loe plaque index,plaque of cervical third of tooth.

\section{Introduction}

Dental plaque is the leading cause of oral diseases, so elimination or reduction of plaque is necessary for prevention of these diseases Oral health is of prime importance to all individuals. Oral hygiene habits are instilled in childhood itself irrespective of the nationality or geographic location of an individual.The most reliable and accepted method of oral hygiene maintenance is mechanical methods of tooth cleaning but adjuvants for decreasing plaque formation and maintaining oral hygiene have been sought ${ }^{1}$.

Dental plaque is the soft deposits that form the biofilm adhering to tooth surface or other hard surface in oral cavity, including removal and fixed restoration ${ }^{2}$.It contains a complex blend of various microorganisms. Plaque is considered as the precursor of dental caries, gingivitis and periodontitis. The prevention and control of dental caries and as well the periodontal disease is dependent on optimal plaque control ${ }^{3}$.Also Based on large epidemiologic studies, dental plaque is the primary etiologic factor in gingival inflammation, the condition that is followed by chronic periodontitis in majority of patients, due to apical extension of supragingival plaque to the subgingival area (Hancock, 1996), which is a favorable ecological niche for the accumulation of anaerobic gram-negative bacteria such as Porphyromonas gingivalis, Tannerella forsythia, Actinobacillus sp., Prevotella sp., and Fusobacterium sp., known as putative periodontal pathogens (Socransky and Haffajee, 2005; Haffajee and Socransky, 2006; Haubek et al., 2004) ${ }^{4}$.

Although dental caries and periodontal disease wouldn't put in risk the patient's life, they are problems of importance in public health, not only because of high prevalence but for the influence among the individuals, related with pain, discomfort, social and function limitations, affecting the hole life of these people ${ }^{5}$.

Interest in alternative toothpaste based on homemade product has increased recently. Among this, coconut oil has received greater attention in many countries.

Coconut oil is edible oil and is consumed as a part of the staple diet in many tropical countries. Coconut oil is highly desired and easily available oil in India. Coconut oil contains $92 \%$ saturated acids, approximately $50 \%$ of which is lauric acid. Human breast milk is the only other naturally occurring substance with such a high concentration of lauric acid. Lauric acid has proven anti-inflammatory effects and antimicrobial effects ${ }^{1}$.

Stevia is a species that produce sweet steviol glycosides. It has been used to sweeten tea for centuries dating back to the Guarani Indians of South America. Glycosides responsible for the plants sweeteners were 
discovered in 1931. Its extracts are used today as a food additive by the Japanese and Brazilians and as a noncaloric sweetener ${ }^{6}$.

Sodium bicarbonate is also known as baking soda For the last two decades, it has been used as a dentifrice abrasive and an oral deodorizer. Sodium fluoride, which provides soluble fluoride, is compatible with sodium bicarbonate ${ }^{7}$.

Thus, an attempt was made to compare the effectiveness of triclosan-based dentifrice and homemade dentifrice by conducting a trial among the children of udaipur city over a period of 7 days.

\section{Materials And Methods}

After ethical approval, sample sizes of 100 subjects aged between 8 to 12 years were selected for the study. Eligible cases met the following inclusion criteria: children between the age group of 8 to 12 years, children with mixed dentition, Caries-free mouth. The criteria for exclusion were presence of fixed orthodontic appliance, history of allergy to toothpastes, defects in the buccal and lingual surfaces of the teeth that may facilitate the retention of dental plaque. The study was designed as a randomized double-blinded clinical trial.The subjects were randomly divided into two groups 50 children in each group were divided. Group 1 (children used homemade toothpaste) and Group 2 (children used commercial toothpaste). Oral examination was performed at the begning of the study (baseline) and after 7 days. Only the plaque of the cervical third of the tooth was evaluated by using disclosing solution.On baseline Plaque index score for both the group were rocorded by using PI score(Silness and Loe). The evaluation of score was done on the entire dentition.

After that children of both the groups were advised to follow the Fones brushing technique by using their toothpaste in front of the clinician for 7 days.After 7 days oral hygine status was reassessed by using Plaque Index (Silness and Loe) with the help of disclosing agent. The use of the appropriate brushing technique, as previously instructed, was stressed upon at each day.

\section{Result}

Statistical analyses was performed using personal computer with SPSS (Version 16). Data comparison was done by applying specific statistical tests to find out the statistical significance of the comparisons. Statistical tests employed for the obtained data in this study were independent t-test and paired sample t-test.All 100 subjects successfully completed the study period of 7 days. In comparison, using independent t-test between the homemade and commercial groups for plaque, no statistically significant difference was obtained at Days 0 and 7. On intra-group comparison using paired t-test at different time periods for plaque index, a significant reduction in plaque scores was obtained in the first week itself (Table 1).

Intra Group (Table 1)

\begin{tabular}{|l|l|l|l|l|l|l|}
\hline Group & Examination day & Mean & S.D & M.D & T-value & P-value \\
\hline \multirow{2}{*}{1} & 0 & 0.3463 & 0.064 & 0.574 & 3.433 & 0.04 \\
\cline { 2 - 4 } & 7 & 0.2889 & 0.2028 & & & \\
\cline { 2 - 5 } 2 & 0 & 0.3200 & 0.4766 & 0.247 & 3.476 & 0.04 \\
\cline { 2 - 4 } & 7 & 0.2953 & 0.4291 & & & \\
\hline
\end{tabular}

$\mathrm{p}<0.05=$ statistically significant, $* * \mathrm{p}<0.01=$ highly significant, $* * * \mathrm{p}<0.001=$ very highly significant.

On intergroup comparison, though the plaque scores were lower in the homemade group at Day 7 . But there was no significant difference seen between the two groups (Table2).

INTER GROUP (Table 2)

\begin{tabular}{|l|l|l|l|l|l|}
\hline Examination day & Group & Mean & S.D & t-value & p-value \\
\hline \multirow{2}{*}{ Baseline } & 1 & 0.3463 & 0.6465 & 1.267 & 0.216 \\
\cline { 2 - 4 } & 2 & 0.3200 & 0.4766 & & 0.522 \\
\cline { 1 - 3 } one week & 1 & 0.2889 & 0.2028 & 0.606 \\
\cline { 2 - 3 } & 2 & 0.2953 & 0.4291 & & \\
\hline
\end{tabular}

$\mathrm{p}<0.05=$ statistically significant, $* * \mathrm{p}<0.01=$ highly significant, $* * * \mathrm{p}<0.001=$ very highly significant.

\section{Discussion}

Microbial plaque growth occurs within hours, and it must be completely removed at least once every 48 hours in the experimental setting with periodontally healthy subjects to prevent inflammation..The principal ingredient in homemade toothpaste used has several medicinal properties. In animal studies coconut oil was found to be an effective burn wound healing agent and this was attributed to its anti-inflammatory and antiseptic properties.Also Oil pulling has been proven to be an effective method in reducing plaque formation and plaque induced gingivitis. So in our homemade toothpaste the principal ingredient was coconut oil.

The difference in mean dental plaque scores between Days 0 to 7 were found to be statistically 
significant ( $\mathrm{p}$ 0.04) in both groups for maxillary and mandibular arches. However the differences between the groups were not statistically significant but plaque reduction was more in homemade tooth paste as compared to commercial.

Our study is in agreement with study by Gayle bunger McCombs et al in 2001 who used Sodium bicarbonate (NaHCO2, baking soda) and salt, separately or in combination, as a dentifrice and concluded that there was a significant reduction in plaque score and safe to oral tissue.

Present study is in agreement with Kerzicnik L, Stendell N, McMuny M and Hagan D in 1998 concluded that stevia can replace some or all of the sugar (sucrose) in recipes without drastically affecting the visual acceptability or physical characteristics of the food product.

Our study is also in agreement with Faizal C. Peedikayil et. al. in 2015 concluded that Oil pulling has been proven to be an effective method in reducing plaque formation and plaque induced gingivitis.

\section{Conclusion}

The result of this study have given useful information about the positive effect of homemade toothpaste on decreasing plaque score.It is suggested that the use of the homemade toothpaste containing coconut could be useful aid to obtaining a significant reduction for plaque index compared to the commercial toothpaste .Within the limited sample size we can conclude homemade toothpaste is definitely an alternative in restoring gingival health for those using commercially available tooth paste.

\section{References}

[1]. Peedikayil.F C, Sreenivasan.P, Narayanan.A . Effect Of Coconut Oil In Plaque Related Gingivitis - A Preliminary Report.J Nigerian Medical 2015;56(2):143-147.

[2]. Newman M.G,Takie H.H,Carranza F.A Textbook Of Clinical Periodontology $9^{\text {th }}$ Edition:1-1032

[3]. Srinivasa.S, Nandlal.B, K. T. Srilatha. A Comparative Evaluation Of A Commercially Available Herbal And Non-Herbal Dentifrice On Dental Plaque And Gingivitis In Children-A Residential School-Based Oral Health Programme. J. Dent. Oral Hyg.2011;3(8):109-113

[4]. Radafshar.G, Mahboob.F, Kazemnejad.E. A Study To Assess The Plaque Inhibitory Action Of Herbal-Based Toothpaste: A Double Blind Controlled Clinical Trial. Journal Of Medicinal Plants Research.2010;4(12):1182-1186.

[5]. Souza Schroeder.MD, Ulema Ribeiro G.L. Evaluation Of Periodontal Index Of Gingival And Plaque With Dental Crowding In Development Of Gingivits In Children And Adolescents.2004;1(1):17-21.

[6]. Kerzicnik L, Stendell N, Mcmuny M And Hagan D, Food Characteristics Of Recipes Using Stevia Sweetner - A Proposed Herbal Sugar Substitute, J Am Dietet Assoc, 1998,

[7]. Dr. Hefferren.J, Dr. Na Li. Dentifrice Abrasives: Heroes Or Villains.2008:1-8.

[8]. Joseph Silverman, Glenn Rosivack, Pamela B. Matheson, Milton I. Houpt Comparison Of Powered And Manual Toothbrushes For Plaque Removal By 4- To 5-Year-Old Children.Journal Of Pediatric Dentistry 2004;26(3):225-230.

[9]. Madlena M, Banoczy J, Gotz G, Marton S, Miklos Kaan Jr,Nagy G Effects Of Amine And Stannous Fluorides On Plaque Accumulation And Gingival Health In Orthodontic Patients Treated With Fixed Appliances: A Pilot Study.J OHDM 2012 ;11(2):57-61.

[10]. Bruce Fife Dental Health With Oil Swishing Evidence That Oil Pulling Eradicates Harmful Bacteria. Well Being Journal 2008:3942. 